\title{
Effect of porosity gradient on the permeability tensor
}

\section{Mahyar Madadi $^{1 *} \quad$ Tobias M. Müller $2,3 *$}

${ }^{1}$ Peter Cook Centre of Carbon Capture and Storage, The University of Melbourne, Parkville, VIC 3010, Australia

${ }^{2}$ School of Earth Sciences and Engineering, Hohai University, Nanjing 211100, China

${ }^{3}$ Department of Seismology, Centro de Investigación Cientí?ca y de Educación Superior de Ensenada (CICESE), 22860 Ensenada, BC, México

\section{Correspondence}

Mahyar Madadi, Peter Cook Centre of Carbon Capture and Storage,

The University of Melbourne, Parkville, VIC 3010,

Australia

Email: mahyar.madadi@unimelb.edu.au

Funding information

This work was supported by CICESE under Project 641174 and CONACYT under grant no. CB2016-286476.
Experimental investigations often reveal that there is a correlation between porosity and permeability in rocks. The most commonly used model to predict the porosity-permeability relation is due to Kozeny-Carman or its many modi? cations. However, these models are problematic because they always involve an empirical constant and, in macroscopically heterogeneous porous media with non-uniform porosity, more than one empirical constant would be required. Moreover, the tensor character of the permeability is not accounted for when the permeability is conceptualized as a plain function of the porosity. To overcome these limitations, we devise an approach by analyzing the drag force in the volume averaging framework of poroelasticity. This allows us to deduce an expression for the inverse permeability tensor. It is the sum of the inverse of the permeability pertaining to a representative volume element and the second spatial derivative of porosity. Therefore, the gradient of porosity changes the permeability depending on the variations of macroscopic porosity variations. This result is thought to be relevant in applications where porosity maps are converted into permeability maps.

KEYWORDS

Porosity-permeability correlations, Analytical solution

\footnotetext{
*Equally contributing authors.
}

This is the author manuscript accepted for publication and has undergone full peer review but has not been through the copyediting, typesetting, pagination and proofreading process, which may lead to differences between this version and the Version of Record. Please cite this article as doi: 10.1111/1365-2478.12922 


\section{1 | INTRODUCTION}

The most fundamental de? ning characteristics of a porous rock are the porosity and permeability (Sahimi, 2011). The porosity is ratio of pore to bulk volume and quanti? ?es how much ? porous matrix. The permeability is the ability for ? ? uids to ?low through the porous matrix. Though porosity and permeability are fundamentally di? erent quantities, they are not independent. There is rich empirical evidence for porosity-permeability relations (Scheidegger, 1974; Schön, 2015). It appears popular to conceptualize these correlations based on the geometrical arrangement of the pores, their inter-connectivity, and the grain size distributions. The perhaps best known is the Kozeny-Carman relation (Schön, 2015). This relation has been adapted and modi? in various contexts borrowing ideas from fractal geometry and percolation theory (Costa, 2006).

As for the origin of porosity-permeability relationships, Scheidegger (1974) distinguishes between hydraulic radius theories and drag force theories. The most prominent hydraulic radius theory has been developed by Kozeny and later extended by Carman. Scheidegger critically remarks that these theories come always with some geometrical constants, which cannot be independently measured, and are thus often overrated. He also critiques that a simple correlation between porosity and permeability is physically not plausible, as it is perfectly possible to have two porous media with the same porosity but di? erent permeability. Another concern refers to the spatial heterogeneity of porosity, possibly with large porosity ? uctuations. The question is then how this porosity ? luctuations a? ?ect the correlation between porosity and permeability. Up to now, there is no theoretical analysis which quanti? es the e? ect of porosity variations on the total permeability of porous media. It has to be noted that Darcy's law is valid in porous structures even if the porosity ? uctuates (Scheidegger, 1974; Ma, 2015). This fact points to a fundamental ?aw in the Kozeny-Carman type theories, which do not capture porosity ?uctuations at macro-scale.

Drag force theories essentially consist in calculating the ? (e.g. spheres). For more complex obstacles Scheidegger sub-classi?]es the drag force approach as the "Stokes ?low approach", which means that the Stokes equation is solved numerically at pore-scale level. While in principle possible, drag force theories and in particular the Stokes ? low approach have not been employed to study the permeability in macroscopically heterogeneous porous materials. Drag force approaches based on ? rst principles have been developed by (Bear and Bachmat, 1967; Whitaker, 1986). Bear and Bachmat (1967) consider microscopic (pore-scale) and macroscopic factors of the medium by using statistical averaging procedures. The predicted permeability tensor is proportional to the statistical average of the tortuosity, another di? cult-to-determine second rank tensor and the hydraulic conductance of micro-channels (Bear, 1988, page 106). Another theoretical framework to study the permeability from ? rst principles is the volume averaging method (Whitaker, 1986; Bear and Cheng, 2010). It yields to the permeability tensor of heterogeneous porous media. However, the predicted permeability is of rather complex form and the geometrical operators are cumbersome rendering any interpretation di? cult or requires heavy computational e? ? ort. Indeed, understanding the correlation of permeability with geometrical parameters of porous media remains a challenging problem(Rezaei Niya and Selvadurai, 2017).

Our aim is to derive an analytical relation between the permeability and porosity in macroscopically heterogeneous porous media, i.e. in the presence of porosity gradients. This paper is organized as follows. First, we lay out a few basic concepts that will bring out the general meaning of the permeability (section 2). In section 3 we use the volume averaging theorem to analyze the drag force interaction in a porous medium conceptualized as a granular medium and deduce an analytical expression for the permeability tensor. In the section 4 , we numerically illustrate and discuss the e? ? ect of porosity gradients leading to a 'heterogeneous' permeability map as opposed to a 'homogeneous' permeability map when there are no porosity-gradients.

This article is protected by copyright. All rights reserved. 


\section{2 | CONCEPTS UNDERPINNING THE DRAG FORCE THEORY}

\section{1 | Permeability, Darcy's law, and concept of RVE}

The permeability tensor $\left(\kappa_{i j}\right)$ and ? luid shear viscosity $\left(\mu^{\mathrm{f}}\right)$ are introduced as proportionality constants in Darcy's law connecting the volume of ? uid traversing a cross section of a porous medium sample per unit time, the so-called ? Itration velocity, due to an applied pressure di? erence across the inlet and outlet. In absence of gravity, the threedimensional form of Darcy's law is written as the partial di? erential equation

$$
\bar{q}_{i}=-\frac{\kappa_{i j}}{\mu_{\mathrm{f}}} \partial_{j} \bar{p}^{\mathrm{f}} .
$$

It is a macroscopic law implying that the ? Itration velocity $\bar{q}_{i}$ and the ? luid pressure $\bar{p}^{f}$ are macroscopic ?elds denoted as such with an overbar. The ? Itration velocity expressed in terms of the ? luid velocity is $\bar{q}_{i}=\eta_{0} \bar{v}_{i}^{f}$ with the porosity $\eta_{0}$, or, when expressed relative to the solid velocity, $\bar{q}_{i}=\eta_{0}\left(\bar{v}_{i}^{f}-\bar{v}_{i}^{\mathrm{s}}\right)$. The permeability tensor is also a macroscopic quantity which means it requires the existence of a representative volume element (RVE) of the porous medium in the same way the porosity becomes only meaningful at the scale of the RVE.

The spatial derivative operator in Eq. (1) is de? ned at macroscopic scale, i.e. the scale of the RVE or larger. This becomes clear when the volume averaging method is used to derive Darcy's law (Whitaker, 1986; Bear and Cheng, 2010). In fact, a derivative operator at macro-scale can be only de?ned in a physically meaningful way if there is a minimum characteristic physical length de? ned at macroscopic level. This means that ?uctuations of physical parameters assume di? erent meaning at di? erent scales. To take into consideration the ?uctuation of a physical property at sub-RVE scale, not only we have to use the volume average pertaining to this sub-RVE scale, also we have to use the derivative operators at that scale. Figure 1 schematically shows the RVE for porosity and permeability. It illustrates the domain where averaging theory, or poroelasticity theory become meaningful. One can assume a characteristic volume, i.e. $\Delta \mathrm{U}_{0 \eta}$ for porosity. It is the minimum volume for which the porosity value is not any longer a? ected by pore-scale ? uctuations. The RVE is not unique for all parameters of a porous medium. This means that if there is a characteristic volume for porosity $\left(\Delta \mathrm{U}_{0 \eta}\right)$, then it will not be the same for permeability $\left(\Delta \mathrm{U}_{0 k}\right)$. This also means that there is not a one-to-one correlation of porosity-permeability even for homogeneous media, unless the ? uctuations of these parameters would be directly proportional to each other. In macroscopically heterogeneous media, where the physical properties vary in space, one can expect that any correlation depends on macroscopic ?uctuations.

\subsection{Drag force in the volume averaging framework of poroelasticity}

Let us consider a porous medium of volume $V$ which is at least of the size of a RVE. In the volume-averaging framework of poroelasticity developed by Sahay et al. (2001) the drag force $\bar{I}_{j}$ emerges when the equations of motion for the solid and ?uid are averaged. In the quasi-static approximation (all acceleration terms neglected) these volume-averaged equations of motion of solid and ? uid phase are

$$
\begin{array}{r}
\partial_{k}\left(\left(1-\eta_{0}\right) \bar{\sigma}_{j k}^{\mathrm{s}}\right)+\bar{I}_{j}=0 \\
\partial_{k}\left(\eta_{0} \bar{\sigma}_{j k}^{\mathrm{f}}\right)-\bar{I}_{j}=0
\end{array}
$$

This article is protected by copyright. All rights reserved. 


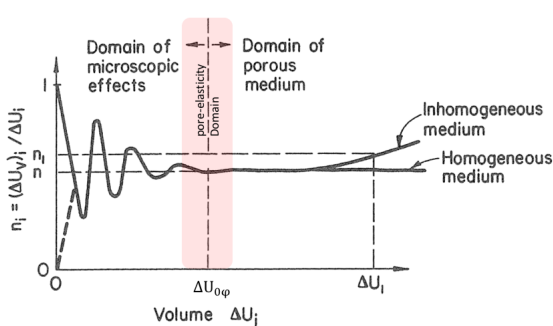

(a)

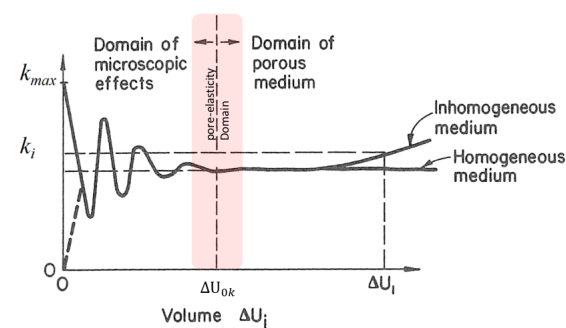

(b)

FIGURE 1 Schematic of the representative volume element (RVE) for porosity (a) and permeability (b). [Adapted after Bear (1988)]

with the macroscopic solid and ? $\mathrm{uid}$ stress tensors $\bar{\sigma}_{j k}^{\mathrm{s}}$ and $\bar{\sigma}_{j k}^{\mathrm{f}}$. The drag force density is represented as surface integral over the solid-? uid interface $A$, i.e. the pore boundaries,

$$
\bar{I}_{j}=\frac{1}{V} \int_{A^{\mathrm{sf}}} \sigma_{j k}^{\mathrm{s}} \hat{n}_{k} d A=-\frac{1}{V} \int_{A^{\mathrm{fs}}} \sigma_{j k}^{\mathrm{f}} \hat{n}_{k} d A
$$

where $A^{\text {sf }}$ is the surface element with the normal vector pointing into the solid phase (Müller and Sahay, 2019). The integrands are the pore-scale stress tensors of the solid and ? uid phase, respectively, That is to say the solid and ?uid tractions $\sigma_{j k}^{\mathrm{s}} \hat{n}_{k}$ and $\sigma_{j k}^{\mathrm{f}} \hat{n}_{k}$ are acting on the pore-interfacial area contained in the averaging volume. They are equal and opposite on account of Newton's third law. Sahay et al. (2001) further deduce that the drag force is proportional to the relative ? ?uid-solid velocity with the permeability tensor as proportionality coe? cient. Thus, its representation in terms of macroscopic ?elds is set up as (Sahay et al., 2001)

$$
\bar{I}_{j}=-\eta_{0}^{2} \mu^{\mathrm{f}}\left(\kappa^{-1}\right)_{j k}\left(\bar{v}_{k}^{\mathrm{s}}-\bar{v}_{k}^{\mathrm{f}}\right)-\bar{p}^{\mathrm{f}} \partial_{j} \eta_{0}
$$

This choice guarantees consistency with Darcy's law. Indeed, by substituting Eq. (5) into (3) we recover Darcy's law (Eq. 1). The porosity gradient term on the right-hand side ensures consistency with Darcy's law even in the presence of porosity gradients, i.e. in macroscpically heterogeneous porous media.

\section{3 | GENERALIZED DRAG FORCE APPROACH}

\section{1 | Decomposition of interfacial area for a granular pack}

We now envisage an idealized porous medium made out of a closed pack of $N_{t}$ grains so that the solid phase is a polydisperse pack. If the ? ? id is taken out of this porous medium, i.e. in the dry case, it is a random polydisperse pack constituting a granular medium. We further assume that the solid-? $A=\Sigma_{\alpha}^{N_{t}} A_{\alpha}$, where the $A_{\alpha}$ is the solid-? ?uid interfacial area of grain $\alpha$. In view of Eq. (4) the drag force becomes

This article is protected by copyright. All rights reserved. 


$$
\bar{I}_{j}=-\frac{1}{V} \sum_{\alpha=1}^{N_{t}} \int_{A_{\alpha}^{\mathrm{fs}}} \sigma_{j k}^{\mathrm{f}} \hat{n}_{k} d A,
$$

where $\int_{A_{\alpha}^{\mathrm{fs}}} \sigma_{j k}^{\mathrm{f}} \hat{n}_{k} d A$ is taken as the ? uid force exerted onto a single grain. As the integral is over all interfacial surfaces, in bounded porous media, we have two type of grains. First, those grains which are part of the outer boundary and which are therefore interacting with the outside ? luid and boundary condition. Second, the grains in the interior of the porous medium (total number of these interior grains is $N_{i n}$ ). Therefore, the drag force (6) can be further decomposed. One component referring to a macroscopically homogeneous medium, $\bar{I}_{j}^{\mathrm{h}}$, and another component that exists due to the presence of macroscopic heterogeneities, $\bar{I}_{j}^{\mathrm{i}}$. Both components can be represented as a sum over grains. To obtain $\bar{I}_{j}^{\mathrm{h}}$ it is su? cient to sum up the contributions within one RVE, as all other poroelastic material points yield to the same value. In contrast, to obtain $\bar{I}_{j}^{i}$ the summation extends beyond the RVE in order to capture the contribution of the macroscopic heterogeneities. In the following we assume that $\bar{I}_{j}^{\mathrm{h}}$ is known and concentrate our analysis on $\bar{I}_{j}^{\mathrm{i}}$. Without loss of generality we represent the drag force as

$$
\bar{I}_{j}=\bar{I}_{j}^{\mathrm{h}}+\bar{I}_{j}^{\mathrm{i}}=\bar{I}_{j}^{\mathrm{h}}-\frac{1}{V} \sum_{\alpha=1}^{N_{i n}} \int_{A_{\alpha}^{\mathrm{fs}}} \sigma_{j k}^{\mathrm{f}} \hat{n}_{k} d A,
$$

where one has to keep in mind that the summation over the number of grains $(\alpha)$ extends beyond the number of grains contained in the RVE.

A grain is also subjected to an inter-granular contact force, because without it the dry porous medium would be mechanically unstable. This inter-granular contact force must be part of the drag force. Thus, we further assume that the total drag force subjected to a single grain is the sum of the drag force applied from homogeneous and isotropic ? ?uid surrounding the grain and the e? ective contact force $\left(\sigma_{j k}^{c}\right)$ from its nearest neighbour grains (see Figure 2 ). Figures 2(a)-(c) show schematically the decomposition of the drag force into a ? uid body force plus the e? ective interaction from the solid matrix, i.e. grain contacts. Thus, for a single grain, we have

$$
\int_{A_{\alpha}^{\mathrm{fs}}} \sigma_{j k}^{\mathrm{f}} \hat{n}_{k} d A \simeq \oint_{A_{\alpha}} \sigma_{j k}^{\mathrm{f}} \hat{n}_{k} d A+\sum_{c=1}^{C_{\alpha}} \int_{A_{\alpha}^{\mathrm{c}}}\left(\sigma_{j k}^{\mathrm{c}}-\sigma_{j k}^{\mathrm{f}}\right) \hat{n}_{k}^{\mathrm{c}} d A
$$

It is important to note that in this approximation the integral in the ?rst term on the right-hand side becomes a closed surface integral enclosing the grain.

\section{2 | Fluid body force at level of single grain}

The ? rst integral in the right-hand side of Eq. (8) is interpreted as a ? uid body force. This means that each grain is subjected to a body force exerted from the ? uid onto the grain. Assuming that the ? uid is a Newtonian ?uid then the pore-scale ? ? uid stress tensor is $\sigma_{j k}^{\mathrm{f}}=p^{\mathrm{f}} \delta_{j k}+\mu_{\mathrm{f}} \dot{\gamma}_{j k}$. It contains the hydrostatic pressure $p^{\mathrm{f}}$ and viscous shear stress, which is the shear viscosity multiplied with the strain rate tensor $\dot{\gamma}_{j k}$. Using the Gauss divergence theorem we obtain for the ?uid body force

This article is protected by copyright. All rights reserved. 


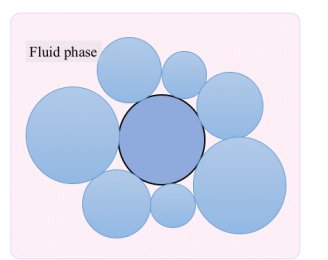

(a)

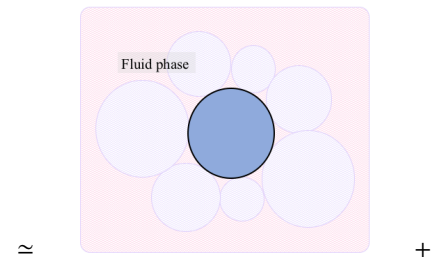

(b)

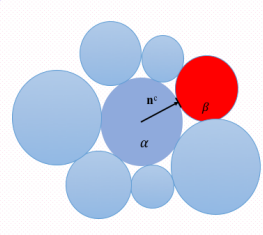

(c)

FIGURE 2 The decomposition of a porous medium (a) into a single inclusion interaction with ?uid (b) and dry granular pack (c).

$$
\oint_{A_{\alpha}} \sigma_{j k}^{\mathrm{f}} \hat{n}_{k} d A=\int_{V_{\alpha}} \partial_{j} p^{\mathrm{f}} d V+\mu_{\mathrm{f}} \int_{V_{\alpha}} \partial_{k} \dot{\gamma}_{j k} d V
$$

This drag force is in general a function of the grain shape. We assume that the grain is a sphere with radius $a_{\alpha}$. We further assume that this sphere is elastically deformable.

The hydrostatic part of ? uid body force is calculated from the pressure ?eld, which only exists in the ?uid space, i.e. outside of the deformable sphere. This is mathematically expressed as $p^{f}(\mathbf{r})=\bar{p}^{f} \Theta\left(\mathbf{r}^{\prime}-\mathbf{r}\right)$, where $r \in V_{\alpha}$, and $\mathbf{r}^{\prime}=a_{\alpha} \hat{r}$; and $\Theta\left(\mathbf{r}^{\prime}-\mathbf{r}\right)$ is the Heaviside function ensuring that the ? uid pressure is de? ned in the ? uid space only. We make use of the equality of derivatives of the Heaviside function $\partial_{j} \Theta\left(\mathbf{r}^{\prime}-\mathbf{r}\right)=-\partial_{j}^{\prime} \Theta\left(\mathbf{r}^{\prime}-\mathbf{r}\right)$, in which $\partial_{j}$ and $\partial_{j}^{\prime}$ are, respectively, the derivative operators with respect to coordinates $r$ and $\mathbf{r}^{\prime}$. Thus, we ?nd

$$
\int_{V_{\alpha}} \partial_{j} p^{\mathrm{f}}(\mathbf{r}) d \mathbf{r}=\int_{V_{\alpha}}-\partial_{j} \bar{p}^{\mathrm{f}} \Theta\left(\mathbf{r}^{\prime}-\mathbf{r}\right) d \mathbf{r}=\bar{p}^{\mathrm{f}} \partial_{j}^{\prime} \int_{V_{\alpha}} \Theta\left(\mathbf{r}^{\prime}-\mathbf{r}\right) d \mathbf{r}=\bar{p}^{\mathrm{f}} \partial_{j}^{\prime} V_{\alpha}
$$

This means that the isotropic part of the ? luid body force is equal to the average of hydrostatic pressure and gradient of grain volume. In the context of soft molecular inclusions, Jackson (1999) shows that the Heaviside function can be replaced by smoother radial distribution functions. Moreover, the variation of solid volume of porous media due to hydrostatic pressure of porous media has been numerically observed (Ahmed et al., 2019).

The second integral in Eq. (9) is the viscous part of the interfacial interaction. It is represented in terms of the strain rate tensor $\dot{\gamma}_{j k}=\frac{1}{2}\left(\partial_{k} \partial_{j} \delta_{k l}+\partial_{k} \partial_{k} \delta_{j l}\right) v_{l}^{f}$. Since the pore-scale ? which can move with its own velocity $v_{l}^{\mathrm{s}}(\mathbf{r})$, we have

$$
\int_{V_{\alpha}} \partial_{k} \dot{\gamma}_{j k} d V=\frac{1}{2} \int_{V_{\alpha}}\left(\partial_{k} \partial_{j} \delta_{k l}+\partial_{k} \partial_{k} \delta_{j l}\right)\left(v_{l}^{f}(\mathbf{r})-v_{l}^{\mathrm{s}}(\mathbf{r})\right) d V
$$

This contribution only exists outside of the deformable sphere. Therefore, as above, we set $v_{l}^{f}(\mathbf{r})-v_{l}^{\mathrm{s}}(\mathbf{r})=\left(v_{l}^{\mathrm{f}}-\right.$ $\left.v_{I}^{s}\right) \Theta\left(r^{\prime}-\mathbf{r}\right)$ and make use of the Heavyside function property to obtain

This article is protected by copyright. All rights reserved. 


$$
\int_{V_{\alpha}} \partial_{k} \dot{\gamma}_{j k} d V=\frac{1}{2}\left(\partial_{k}^{\prime} \partial_{j}^{\prime} \delta_{k l}+\partial_{k}^{\prime} \partial_{k}^{\prime} \delta_{j l}\right) \int_{V_{\alpha}}\left(v_{l}^{f}-v_{l}^{s}\right) \Theta\left(\mathbf{r}-\mathbf{r}^{\prime}\right) d V
$$

Since the average of the relative velocity of the ? uid to volume is $\frac{1}{V_{\alpha}} \int_{V_{\alpha}}\left(v_{l}^{f}-v_{l}^{s}\right) \Theta\left(\mathbf{r}-\mathbf{r}^{\prime}\right) d V=\left(\bar{v}_{l}^{f}-\bar{v}_{l}^{s}\right)$ the viscous part of the ? ?uid body force becomes

$$
\int_{V_{\alpha}} \partial_{k} \dot{\gamma}_{j k} d V=\frac{1}{2}\left(\bar{v}_{l}^{f}-\bar{v}_{l}^{s}\right)\left(\partial_{k}^{\prime} \partial_{j}^{\prime} \delta_{k l}+\partial_{k}^{\prime} \partial_{k}^{\prime} \delta_{j l}\right) V_{\alpha}
$$

It is important to mention that these derivative operators $\left(\partial_{k}\right.$ and $\left.\partial_{k}^{\prime}\right)$ are acting at di? erent physical scales. By shifting from one to another, we are taking into account the variation of the physical quantities at di? erent scales. In other words, the shifting connects the variations of sub-RVE with RVE properties.

\section{3 | The ? ? uid body force for the assembly of grains}

In view of Eqs. (6), (12) and (15) the interior ?

$$
\bar{I}_{j}^{\mathrm{i}}=-\frac{1}{V} \sum_{\alpha=1}^{N} \oint_{A_{\alpha}} \sigma_{j k}^{f} \hat{n}_{k} d A=\bar{p}_{f} \partial_{j}\left(\sum_{\alpha=1}^{N} \frac{V_{\alpha}}{V}\right)+\frac{1}{2} \mu_{\mathrm{f}}\left(\bar{v}_{l}^{s}-\bar{v}_{l}^{f}\right)\left(\partial_{k} \partial_{j} \delta_{k l}+\partial_{k} \partial_{k} \delta_{j l}\right)\left(\sum_{\alpha=1}^{N} \frac{V_{\alpha}}{V}\right) .
$$

Since $1-1 / V \sum_{\alpha=1}^{N} V_{\alpha}$ is the porosity, this expression is re-written as

$$
\bar{I}_{j}^{\mathrm{i}}=-\bar{p}_{f} \partial_{j} \eta_{0}-\frac{1}{2} \mu_{\mathrm{f}}\left(\bar{v}_{l}^{s}-\bar{v}_{l}^{f}\right)\left(\partial_{k} \partial_{j} \delta_{k l}+\partial_{k} \partial_{k} \delta_{j l}\right) \eta_{0}
$$

We observe that in macroscopically inhomogeneous porous media, in which the porosity becomes a function of space, $\eta_{0}(\mathbf{r})$, porosity gradients enter the description of the ?uid body force. The variation of porosity is an integral part in poroelasticity (Ahmed et al., 2019) and the ?rst term shows the consistency with poroelasticity theory.

\subsection{Porosity gradient contribution to the permeability tensor}

The hydrostatic part of the drag force (15) is proportional to the variation of porosity in a macroscopically heterogeneous porous medium. In a homogeneous porous medium this term vanishes. The existence of this term has been postulated by Sahay et al. (2001). Our analysis reveals that this term enters through the synthesis of the e? ective hydrostatic pressure force applied to the grain surface for an assembly of grains. The second term in Eq. (15) represents the drag force due to the motion of a Newtonian ? luid around the grains. We also observe that this term vanishes in a homogeneous porous medium, as then $\partial_{i} \partial_{j} \eta_{0}=0$. This part of the drag force is proportional to the ? Itration velocity. Darcy's law (Eq. 1) suggests that the ?tration velocity is proportional to pressure gradient and the proportionality coe? cient contains the permeability tensor. Thus by comparison with Darcy's law we ?nd that this second term of the drag force is is a tensor of rank two, which is proportional to the variations of inverse of the permeability tensor, $\delta\left(\kappa_{j l}\right)^{-1} \propto\left(\partial_{k} \partial_{j} \delta_{k l}+\partial_{k} \partial_{k} \delta_{j l}\right) \eta_{0}$. Yet, even in the absence of macroscopic heterogeneities, i.e.

This article is protected by copyright. All rights reserved. 
in a macroscopically homogeneous porous medium, there is a permeability tensor assigned to a porous medium via Darcy's law. This permeability is solely described by the pore-scale features and is fully determined at the level of a RVE. We label this permeability as $\kappa_{j k}^{\text {h }}$. Taken altogether, we infer that the inverse of the permeability tensor of a macroscopically heterogeneous porous medium has the representation

$$
\left(\kappa_{j k}\right)^{-1}=\underbrace{\left(\kappa_{j k}^{\mathrm{h}}\right)^{-1}}_{\text {RVE-scale contribution }}+\underbrace{\frac{1}{\eta_{0}^{2}}\left(\partial_{j} \partial_{k}+\partial_{l} \partial_{l} \delta_{j k}\right) \eta_{0}}_{\text {macro-scale heterogeneity contribution }} .
$$

Eq. (16) can be converted into an equation for the permeability tensor. Noting that $\left(\kappa_{i j}\right)^{-1} \kappa_{j k}=\delta_{i k}$ we obtain

$$
\kappa_{i l}\left(\frac{1}{\kappa_{l j}^{\mathrm{h}}}+\frac{1}{\eta_{0}^{2}}\left(\partial_{l} \partial_{j}+\partial_{k} \partial_{k} \delta_{l j}\right) \eta_{0}\right)=\delta_{i j}
$$

Eq. (17) is partial di? erential equation for the porosity variable in which the permeability tensor appears as coef?]cient. This is the main result of this paper. The solution of this equation can be conceived as a porosity-permeability relation. That is to say the permeability tensor components for a macroscopically inhomogeneous porous medium are obtained by solving Eq. (17) and only in the limit of a macroscopically homogeneous porous medium, for which the porosity is constant, one has $\kappa_{j k}=\kappa_{j k}^{\mathrm{h}}$.

\section{4 | NUMERICAL TEST: FROM POROSITY MAP TO HETEROGENEOUS PER- MEABILITY MAP}

To understand the meaning of the porosity-permeability equation (17) we propose a set of numerical tests in which we evaluate a heterogeneous permeability map from a given porosity map. By heterogeneous permeability map we refer to a permeability map that accounts for the macroscopic porosity variations quanti? right-hand side of Eq. (16). If these variations are neglected then we will use the notion of homogeneous permeability map. In these numerical tests, the resulting permeability map will be subjected to scale dependency questions, i.e. whether the averaging volume is of the size of a RVE or not (see also Figure 1). The porosity-permeability equation (17) is deemed to be valid either at the sub-RVE scale and for macroscopically heterogeneous media. However, the minimum size of a RVE is not known a priori. To avoid any deviation from the main focus of this paper, we devise the numerical test based on a known, analytical porosity-permeability relation for a porous medium realized as randomly distributed spherical inclusions.

\subsection{D porosity maps and associated homogeneous permeability maps}

We start with a random sphere pack. The spheres have a radius equal to $50 \mu$-meter. We set the target porosity 0.4 and randomly distribute these spheres, thereby obtaining a binary porous medium realisation. Figure 3(a) shows an ensemble of randomly distributed spherical inclusions. We assume that the local volume fraction of a single spherical inclusion, i.e. the local porosity is $\eta_{0}=0.4$. Figure $3(b)$ shows a $1000 \times 1000$ subset of the ensemble. Then we use the moving window average and create di? erent representations of continuous porosity maps at di? erent length scales.

This article is protected by copyright. All rights reserved. 


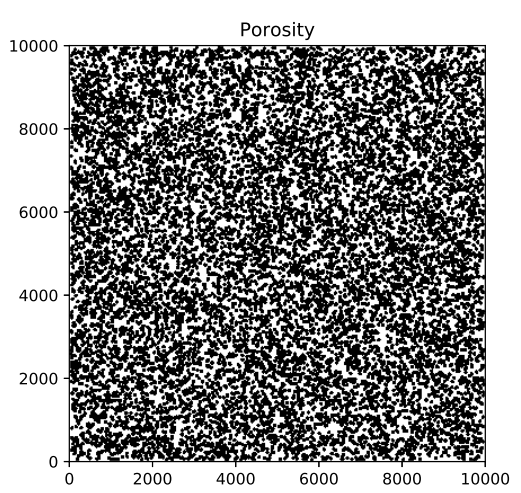

(a)

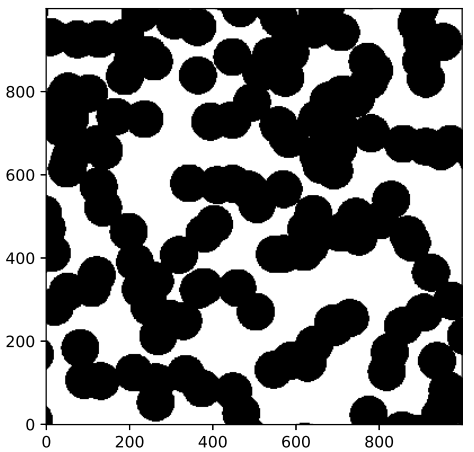

(b)

FIGURE 3 (a) random ensemble of spherical inclusions with target porosity $40 \%$. (b) $1000 \times 1000$ subset of the ensemble.

Figures 4a, 5a and 6a show the continuous porosity maps resulting from a $200 \times 200,1000 \times 1000$ and $5000 \times 5000$ averaging window, respectively.

Then, we create a homogeneous, scalar permeability map by assigning an e? $\mathrm{ective}$, scalar permeability value to each e? ective volume surrounding an inclusion, (i.e. a spherical inclusion in e? ective medium as shown in Figure 2(b)). To do so, we use the analytical permeability prediction for spherical pack presented by Scheidegger (1974). It reads

$$
\kappa=\frac{3}{16} \frac{\eta_{0} r^{2}}{1-\eta_{0}}\left(\frac{2}{4}+O\left(\delta q \rho /\left(\mu \eta_{0}\right)\right)\right)
$$

The porosity and radius of grains are, respectively, $\eta_{0}$ and $r$. The second term $O\left(\delta q \rho /\left(\mu \eta_{0}\right)\right)$ accounts for non-Darcian ? created by moving window averaging. For the homogenous permeability map, we use the harmonic averaging. That means, in each window, we use hamonic averaging of the permeability. These homogeneous permeability maps are shown in Figures $4 b, 5 b$ and $6 \mathrm{~b}$, where the permeability value is given in unit $m^{2}$. We note that another option for calculating the homogeneous permeability map would be to use the $\mathrm{KC}$ relationship or any other porosity-permeability correlation. We use this analytical prediction in order to avoid any systematic error.

\subsection{Heterogeneous permeability maps and the tensorial character of the permeability}

Having constructed continuous porosity maps (Figures $4 \mathrm{a}, 5 \mathrm{a}$ and $6 \mathrm{a}$ ) and the corresponding homogeneous permeability maps (Figures $4 \mathrm{~b}, 5 \mathrm{~b}$ and $6 \mathrm{~b}$ ) we now use Eq. (17) to construct heterogeneous permeability maps. This heterogeneous permeability map takes into account the porosity variations. It is also important to note that Eq. (17) allows us to analyze the tensorial character of the permeability. This is not possible with any porosity-permeability relation of the KC-type which always yields a scalar permeability value only. Figures 7-9 show the heterogeneous permeability maps for the three sizes of the moving window average (these are $200 \times 200,1000 \times 1000$ and $5000 \times 5000$ ). In these Figures, from left to right, the permeability tensor elements are shown, i.e. (a) $\kappa_{x x}$, (b) $\kappa_{y y}$ and (c) $\kappa_{x y}$. The 


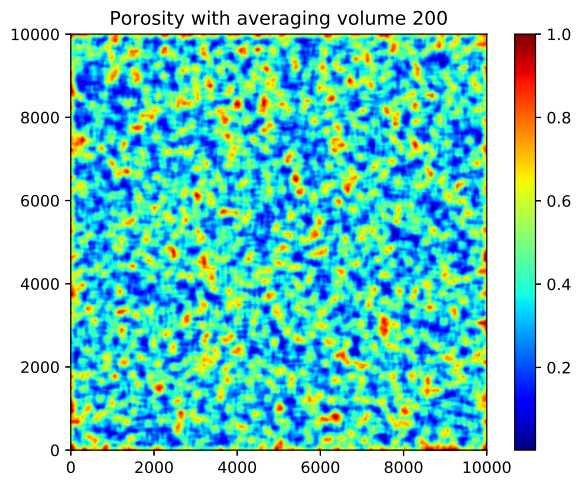

(a)

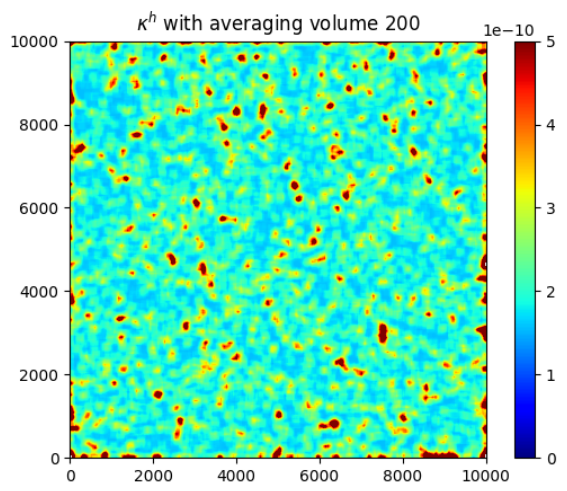

(b)

FIGURE 4 Upscaled porosity (a) and permeability (b) obtained by moving window averaging with a window size of $200 \times 200$.

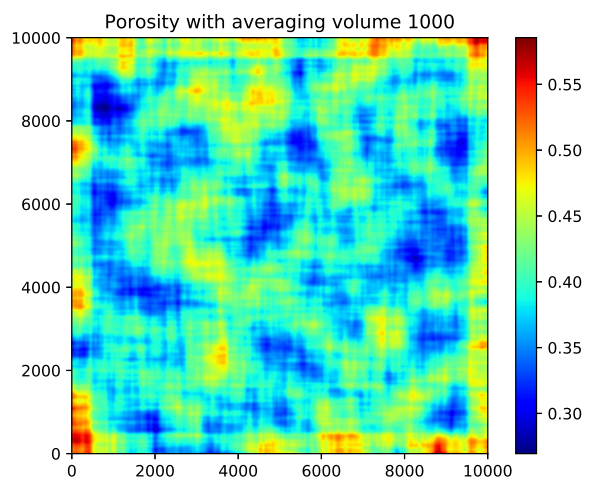

(a)

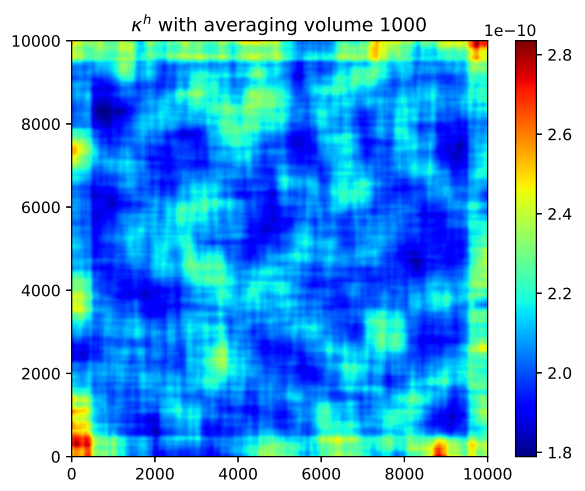

(b)

FIGURE 5 Same as ?gure 4 except for the window size $1000 \times 1000$. 


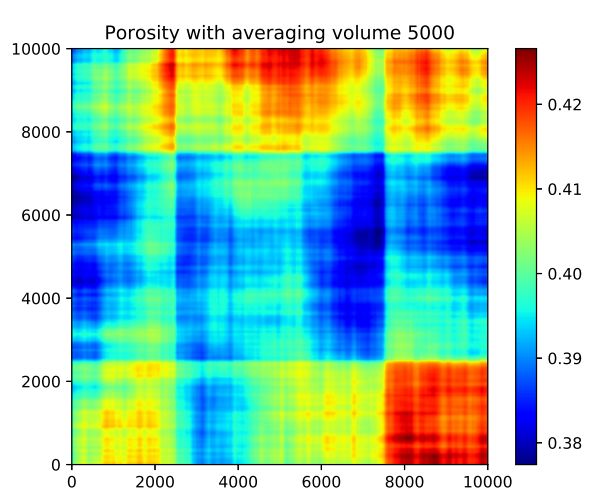

(a)

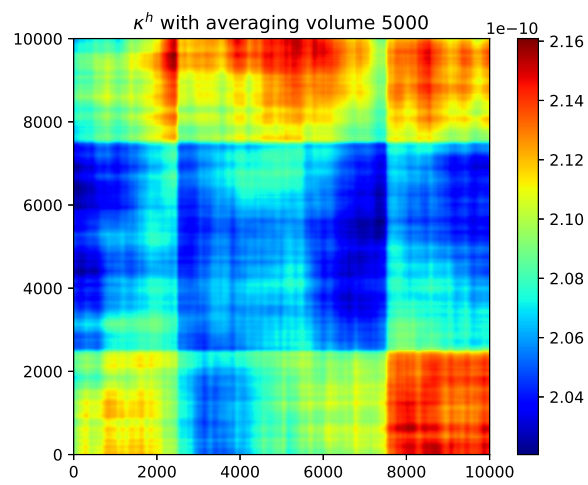

(b)

FIGURE 6 Same as ?gures 5 and 4 except for window size $(5000 \times 5000)$.

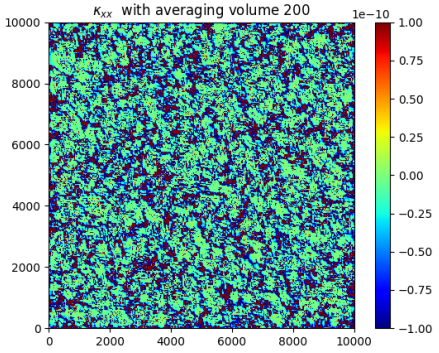

(a)

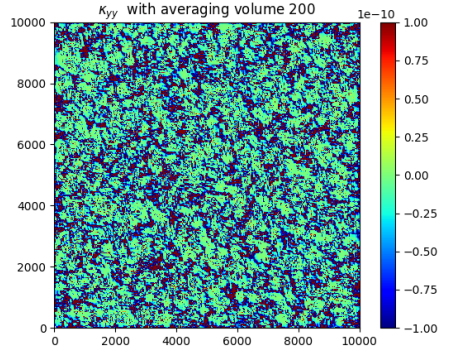

(b)

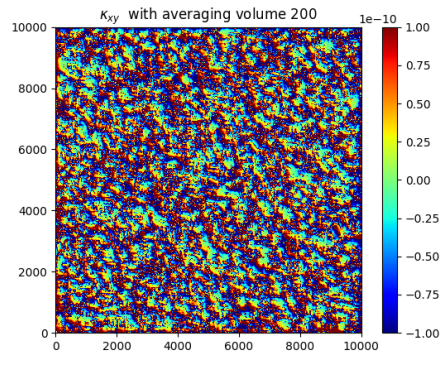

(c)

FIGURE 7 Heterogeneous permeability map. The permeability tensor components are respectively, $\kappa_{x x}, \kappa_{y y}$ and $\kappa_{x y}$. The size of the averaging window is $200 \times 200$.

permeability values are in unit of $m^{2}$.

Comparing these heterogeneous permeability maps with the homogeneous maps we make the following observations. For the $k_{x y}$ component we note that the spatial variations of porosity do not appear to matter. This is expected since there are no obvious spatial alignments in the porosity maps which would render a heterogeneous $\kappa_{x y}$ distribution. We further observe that the $\kappa_{x x}$ and $\kappa_{y y}$ components are virtually equal. This is expected for precisely the same reason: The original porosity maps are isotropic and hence no preferred direction for ? low should be generated and hence $\kappa_{x x}(\mathbf{r})=\kappa_{y y}(\mathbf{r})$. Yet, the permeability values are di? erent if compared with their respective homogeneous permeability map. This di? erence is due to the presence of porosity ? uctuations. A direct comparison of the homogenous and heterogeneous permeabilities for a 1D cross section taken at $y=5000 \mathrm{~m}$ is shown in Figure 10. This means that macroscopic porosity ? uctuations, depending on their spatial arrangement, either strengthen or weaken the ability to ?ow. These local ? luid pathway alterations could not be detected when a KC-type porosity-permeability relation is used to generate a permeability map from a given porosity map.

We note that in these numerical examples the porosity is a smoothly varying ?eld. Correspondingly the porosity

This article is protected by copyright. All rights reserved. 


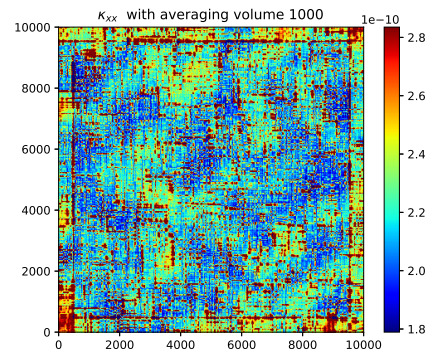

(a)

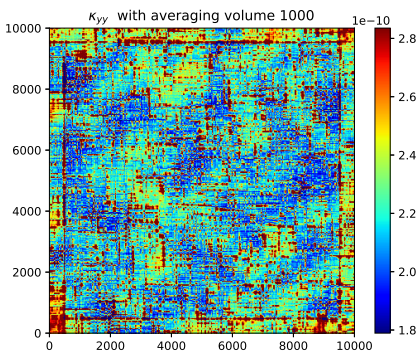

(b)

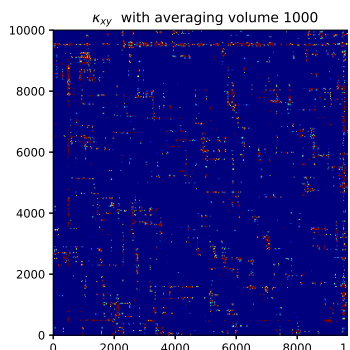

(c)

FIGURE 8 Same as Figure 7 except for the size of the averaging window, which is $1000 \times 1000$.

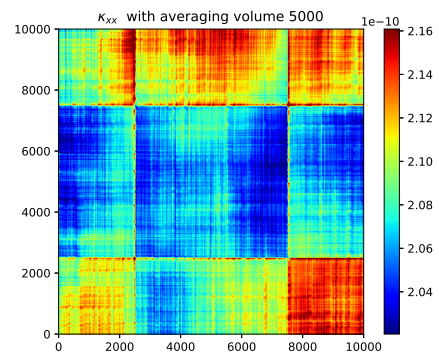

(a)

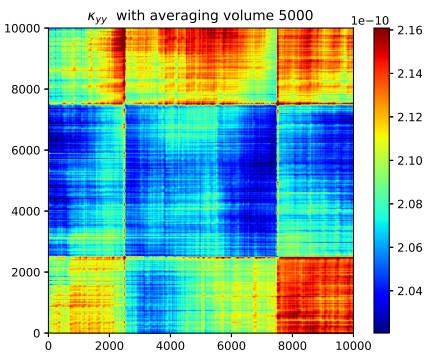

(b)

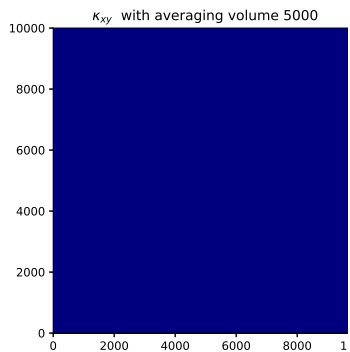

(c)

FIGURE 9 Same as Figure 7 except for the size of the averaging window, which is $5000 \times 5000$.

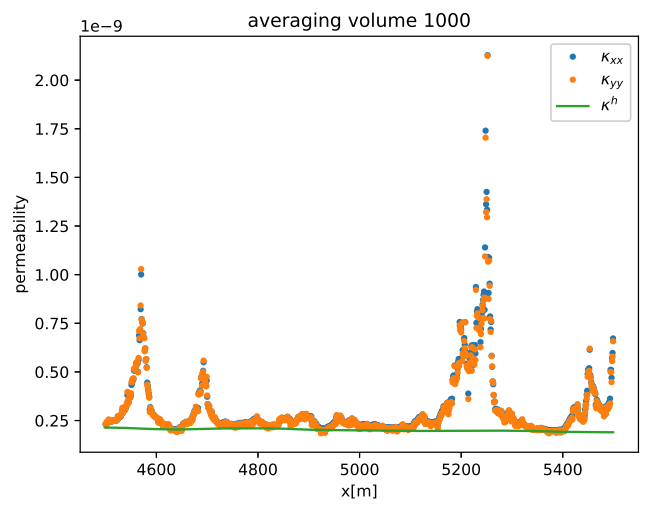

(a)

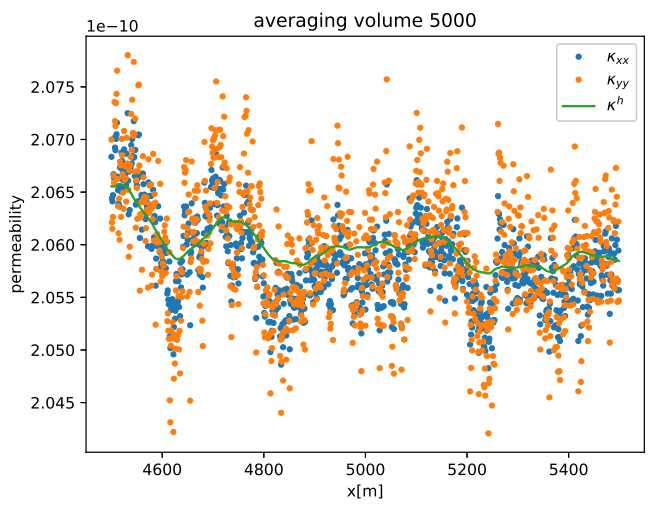

(b)

FIGURE 10 1D cross section showing the ?uctuations of the heterogeneous permeability versus the homogeneous permeability at $y=5000$ for di? erent $x$ coordinate for the averaging window (a) $1000 \times 1000$ and (b) $5000 \times 5000$. 
gradients are moderate and the incurring correction to the permeability is relatively small. However, in real geological structures porosity gradients can become strong. Fractured porous rock masses would be a prime example (Olson et al., 2009; Gale et al., 2014). In such circumstances we anticipate that the heterogeneous permeability can be substantially di? erent from the homogeneous permeability.

\section{5 | CONCLUSION}

We have developed a drag force approach to determine the permeability tensor in macroscopically heterogeneous porous media. The notion of macroscopic heterogeneity implies that the porosity is varying in space. The construction principle is based on the empirical fact that Darcy's law remains its validity in macroscopically heterogeneous porous media. By comparison with the area integral representing the drag force in the volume averaging theory of poroelasticity and making a single solid inclusion approximation we derive a partial di? erential equation for the the porosity variable in which the permeability tensor appears as a coe? cient. We therefore conclude that in macroscopically heterogeneous porous media, the porosity-permeability relation is not simply a complex function of the porosity as previously thought. Instead, our result suggests that the permeability depends on the gradient of the porosity and how this gradient varies in space. We conjecture that the deduced partial di? erential equation for the permeability tensor provides a new avenue to convert porosity maps into maps for the permeability tensor components.

\section{Acknowledgements}

We would like thank Prof. Muhammad Sahimi and Prof. Pratap Sahay for their valuable and critical comments on this work. MM would like appreciate Prof. Stephan Matthai for his support to present this work at 5th International Workshop Rock Physics at Hong Kong. This work was supported by CICESE under Project 641174 and CONACyT under Grant CB2016-286476.

\section{Data availability statement}

The data that support the ?ndings of this study are available from the corresponding author upon reasonable request.

This article is protected by copyright. All rights reserved. 


\section{List of Figures}

1 Schematic of the representative volume element (RVE) for porosity (a) and permeability (b). [Adapted

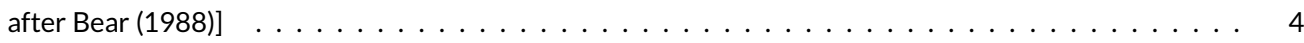

2 The decomposition of a porous medium (a) into a single inclusion interaction with ? luid (b) and dry

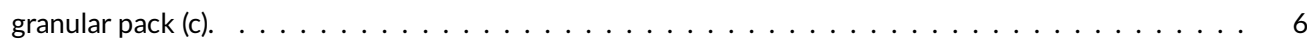

3 (a) random ensemble of spherical inclusions with target porosity $40 \%$. (b) $1000 \times 1000$ subset of the

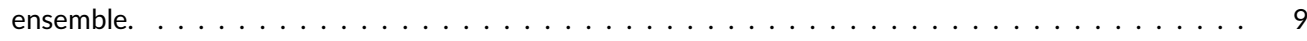

4 Upscaled porosity (a) and permeability (b) obtained by moving window averaging with a window size

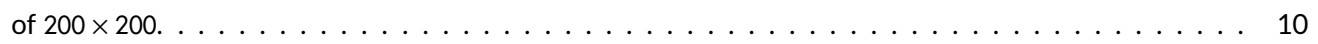

5 Same as ?gure 4 except for the window size $1000 \times 1000 \ldots \ldots \ldots$. . . . . . . . . . . . . . . . . 10

6 Same as ?gures 5 and 4 except for window size $(5000 \times 5000)$. . . . . . . . . . . . . . . . . . 11

7 Heterogeneous permeability map. The permeability tensor components are respectively, $\kappa_{x x}, \kappa_{y y}$ and $\kappa_{x y}$. The size of the averaging window is $200 \times 200 \ldots \ldots \ldots \ldots \ldots \ldots$

8 Same as Figure 7 except for the size of the averaging window, which is $1000 \times 1000 \ldots \ldots \ldots 12$

9 Same as Figure 7 except for the size of the averaging window, which is $5000 \times 5000 \ldots \ldots$. . . . . . 12

$101 \mathrm{D}$ cross section showing the ? luctuations of the heterogeneous permeability versus the homogeneous permeability at $y=5000$ for di? erent $x$ coordinate for the averaging window (a) $1000 \times 1000$ and (b)

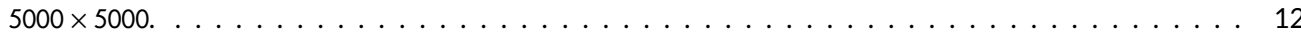

This article is protected by copyright. All rights reserved. 


\section{references}

Ahmed, S., Müller, T. M., Madadi, M. and Calo, V. (2019) Drained pore modulus and biot coe? cient from pore-scale digital rock simulations. International Journal of Rock Mechanics and Mining Sciences, 114, 62 - 70.

Bear, J. (1988) Dynamics of Fluids in Porous Media. Dover Civil and Mechanical Engineering Series. Dover.

Bear, J. and Bachmat, Y. (1967) A generalized theory on hydrodynamic dispersion in porous media. Int. Union Geod. Geophys. Publ., 72.

Bear, J. and Cheng, A. (2010) Modeling Groundwater Flow and Contaminant Transport. Theory and Applications of Transport in Porous Media. Springer Netherlands.

Costa, A. (2006) Permeability-porosity relationship: a reexamination of the kozeny-carman equation based on a fractal porespace geometry assumption. Geophysical Research Letters, 33 (L02318), 1 - 5. Publisher: American Geophysical Union.

Gale, J., Laubach, S., Olson, J., Eichhubl, P. and Fall, A. (2014) Natural fractures in shale: A review and new observations. AAPG Bulletin, 98, 2165-2216.

Jackson, J. D. (1999) Classical electrodynamics. New York, NY: Wiley, 3rd ed. edn.

Ma, J. (2015) Review of permeability evolution model for fractured porous media. Journal of Rock Mechanics and Geotechnical Engineering, 7, 351 - 357.

Müller, T. M. and Sahay, P. N. (2019) Elastic potential energy in linear poroelasticity. GEOPHYSICS, 84, W1-W20.

Olson, J., Laubach, S. and Lander, R. (2009) Natural fracture characterization in tight gas sandstones: Integrating mechanics and diagenesis. AAPG Bulletin, 93, 1535-1549.

Rezaei Niya, S. M. and Selvadurai, A. P. S. (2017) The estimation of permeability of a porous medium with a generalized pore structure by geometry identi? cation. Physics of Fluids, 29, 037101.

Sahay, P. N., Spanos, T. J. T. T. and De La Cruz, V. (2001) Seismic wave propagation in inhomogeneous and anisotropic porous media. Geophysical Journal International, 145, 209-222.

Sahimi, M. (2011) Flow and transport in porous media and fractured rock : from classical methods to modern approaches. Wiley$\mathrm{VCH}, 2 \mathrm{nd}$ edn.

Scheidegger, A. (1974) The physics of ? low through porous media. University of Toronto Press.

Schön, J. (2015) Physical Properties of Rocks: Fundamentals and Principles of Petrophysics. Developments in Petroleum Science. Elsevier Science.

Whitaker, S. (1986) Flow in porous media i: A theoretical derivation of darcy's law. Transport in Porous Media, 1, 3-25.

This article is protected by copyright. All rights reserved. 


\section{University Library}

\section{- M M I E R R A A gateway to Melbourne's research publications}

Minerva Access is the Institutional Repository of The University of Melbourne

Author/s:

Madadi, M;Mueller, TM

Title:

Effect of porosity gradient on the permeability tensor

Date:

2020-01-09

Citation:

Madadi, M. \& Mueller, T. M. (2020). Effect of porosity gradient on the

permeability tensor. GEOPHYSICAL PROSPECTING, 69 (3), pp.542-551. https:// doi.org/10.1111/1365-2478.12922.

Persistent Link:

http://hdl.handle.net/11343/275248 